\title{
INFRASTRUCTURE MANAGEMENT METHODOLOGIES IN RISK SITUATIONS
}

\author{
J.R. MARQUES \& M. DA CONCEIÇÃO CUNHA \\ Department of Civil Engineering, Coimbra University, Portugal.
}

\begin{abstract}
Water supply systems (WSS) are complex but vitally important infrastructures in today's societies. They are exposed to various risk situations from natural disasters and human failings, but criminal acts can also compromise their operations and cause substantial economic and social damage. Several publications on how to tackle these concerns have appeared in the last few years. This paper presents the state of the art in terms of the vulnerabilities, risks, interdependencies and optimization models inherent to WSSs. It further describes the robust optimization method used in engineering systems and in actual WSSs. Finally, the ways in which robustness is included, as described in case studies, are systematized and some conclusions are drawn.
\end{abstract}

Keywords: risk, robust optimization, vulnerability, water supply systems.

\section{INTRODUCTION}

Water supply systems (WSS) are vital infrastructures for society as they provide people with high urban living standards. They are thus regarded as one of the most important pieces of critical infrastructures in the United States [1].

It is now extremely necessary to guarantee the quality and availability of water, to protect public health, to comply with increasingly strict legislation and also because citizens are becoming more aware of its importance. In addition there is a growing need to ensure the economic sustainability of the sector at a time when scarce financial resources mean that interventions have to be ever more judicious, and certainly optimized.

The systems are spread over large areas and connected with one another and with other infrastructures like the electricity grid, telecommunication networks and wastewater drainage systems. They are controlled by a great number of operators and have countless users. All these factors make them vulnerable and studies should be conducted to find ways of identifying and mitigating possible threats to them that could jeopardize their safety and uninterrupted operation.

This paper presents the state of the art in terms of the vulnerabilities, risks, interdependencies and optimization models inherent to WSSs. It further describes the robust optimization method used in engineering systems and in actual WSSs. Finally, the ways in which robustness is included, as described in case studies, are systematized and some conclusions are drawn.

\section{VULNERABILITIES}

WSSs are highly vulnerable infrastructures. Haimes [2] believes that the main challenge that science has to tackle in the design and operation of infrastructure in this third millennium is the development of tools and technologies that can keep facilities such as these in operation. He feels that societies' living standards have improved considerably, but even so the provision of services relies to a great extent on infrastructure, and the risks and vulnerability to failure during operation have grown.

Danneels and Finley [3] see the events of 11 September as the main driving force for the analysis of the vulnerability of infrastructures. In the wake of this attack the USA passed a law on public health security that forced WSSs which serve more than 3,300 consumers to be assessed for vulnerability and risk. The RAM-WTM (Risk Assessment Methodology for Water Utilities) was thus 
developed. This can specify the measures to be implemented to lessen vulnerabilities and thereby increase the security of infrastructure components.

Haimes et al. [4] observe that WSSs vulnerabilities have to be assessed not just for a particular point in time, but for their entire working life, since their nature and level of vulnerability are changing all the time. The authors paid special heed to terrorist attacks since there is a vast amount of literature about natural disasters and relative little about terrorist attacks. They divide possible terrorist threats on WSSs into physical, chemical/biological and cyber threats.

According to Matalas [5] the systems vulnerability can be reduced by implementing security measures such as increasing redundancy, robustness and system resilience. The author also says that there has been little experience in dealing with terrorist attacks compared with natural disasters, and that they tend to be isolated incidents with unforeseeable consequences. Any decisions on preventing such events are taken in a state of complete uncertainty.

Vieira et al. [6] observe that there are a great number of extreme situations that can affect WSSs, they break them down into natural and human events; these may then be subdivided into technological and human failures, and terrorist acts (Fig. 1).

An action that could cause immense damage to public health is the chemical contamination of WSSs. Meinhardt [7] notes that deliberate contamination of WSSs by terrorists could have very serious

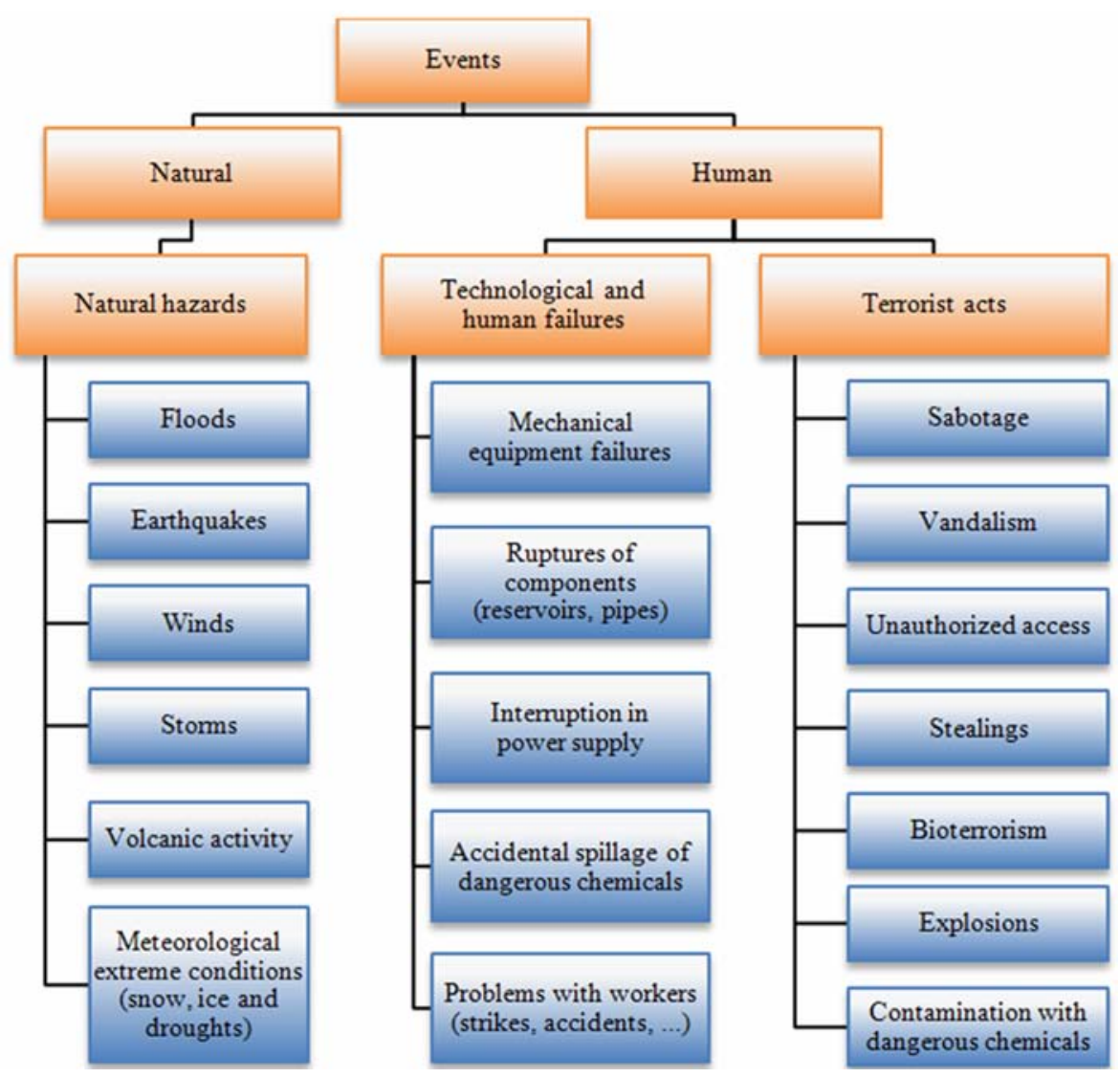

Figure 1: Events that can affect water supply systems. Adapted from [6]. 
consequences because most staffs operating the equipment do not have the sort of knowledge to help them recognize and assess possible water contamination, so the author provides information to enable them to recognize, control and prevent bioterrorist attacks. This article also gives a detailed description of the biological agents that can contaminate the systems, their origins and how to disperse them.

In terms of natural disasters, ICE [8] describes a huge number of disasters and their harmful impact on WSSs. In relation to this aspect of vulnerabilities, WHO [9] reports that failure to consider possible extreme unexpected events during the conception, design, construction and operation stages of systems causes many problems that could be avoided if all such events were contemplated a priori in the decision process. According to this reference the determination and quantification of systems' vulnerabilities can be reduced to the following basic objectives:

- Identifying and quantifying the disasters of natural or human origin that can affect the systems.

- Estimating the susceptibility to damage of components essential in providing water when a disaster has occurred.

- Defining measures to be included in mitigation plans, such as refurbishment projects to lessen the physical vulnerability of system components.

- Identifying measures for an emergency plan so as to guide water companies when emergencies arise.

- Assessing the effectiveness of the mitigation measures and emergency plans and implementing training actions like simulations, seminars and workshops.

\section{RISKS}

The risk of WSSs to different disasters can be calculated based on the probability of a disaster occurring and its likely consequences. But there are still unforeseeable events whose probabilistic distribution cannot be determined. Several ways of structuring methods to manage risk and uncertainty in systems have emerged recently. One is the risk wheel outlined by PLANAT - National Platform on Natural Hazards (risk wheel OFPP [10]).

Ezell et al. [11] describe a method of including risk probability analysis in WSSs called 'Infrastructure Risk Analysis Model' (IRAM). It gives the average values for calculating the critical measures relevant and actually required for the assignment of resources to enhance the system's security. This method has four stages: first, the risks are identified; second, possible scenarios are worked out; third, the system's security is established and expected losses for each scenario calculated; fourth, alternative damage mitigation measures are developed, the risk model is reappraised and the Pareto boundaries of the mitigation alternatives determined. In another paper [12], the method is applied to a WSS to show how it can be used to assess the risk and determine the use of scarce resources to improve the security of the system.

In the context of terrorist attacks, Tidwell et al. [13] describe a model based on latent effects, i.e. events, occurrences, conditions or behaviors that do not necessarily cause an immediate problem but which do so when subsequently combined with other occurrences, conditions or behaviors. These are pinpointed by breaking down the threat into different decision levels and then it is easier to see the basic events that contribute to characterizing the threat. The probabilities of success or failure at the decision levels are then quantified so as to find the probability of the threat actually occurring.

Kunreuther et al. [14] proposed a method that uses cost-benefit analysis to assess the various damage mitigation measures applied to critical infrastructures subject to earthquakes. A systematic assessment and decision procedure comprising the following steps is used:

- Specifying the nature of the problem, alternative options and interested parties.

- Determining the direct costs of the mitigation alternatives. 
- Determining the losses with and without mitigation alternatives.

- Calculating the attractiveness of the mitigation alternatives and choosing the best of them.

The authors use this method with a water distribution system; four possible seismic scenarios are considered, and two alternatives: keeping the system unchanged and strengthening all the system's reservoirs to be able to withstand severe seismic events.

Chang [15] published a paper on the application of a life-cycle cost analysis (LCCA) usually employed to manage infrastructures, and in this case extended to assess the costs and benefits of seismic disaster mitigation for critical infrastructures. As most studies in this area use cost-benefit analyses that do not consider the inevitable wear and tear of infrastructure's component in the course of its working life the author uses LCCA, which implicitly considers this factor. This analysis considers both the costs/benefits of seismic mitigations and the benefits of these actions in reducing maintenance expenses.

\section{INTERDEPENDENCIES}

WSSs cannot be regarded independently when we are looking at their vulnerabilities or reasons for operating failures. The systems depend on other infrastructure such as the electricity grid and telecommunication networks.

Haimes [16] notes that a new dimension of vulnerabilities has emerged in the wake of the rapid spread and integration of telecommunication and computer processes in the management and operation of systems that link the infrastructure to one another in a complex network of interdependencies. It is therefore necessary to quantify them so as to assess and deal with potential threats.

Haimes and Horowitz [17] describe a methodology based on Hierarchical Holographic Modeling (HHM) in a study on infrastructure interdependencies.

Haimes and Jiang [18] developed the Leontief-based infrastructure input-output model that can quantify internal and external links between infrastructures. This model considers critical infrastructures connected to one another and globally as a complex and finds the risks of its subsystems suffering operation failure. It can be implemented in an optimization model, taking the reduction of the risk of operating failure as the objective function and the pre-established amount of resources to be used as the main restriction.

Osorio et al. [19] devised a method to analyze the interdependencies between an electricity grid and a WSS. In it the response of the networks to disruption is measured in terms of the effect that the removal of an element from the network has on overall connectivity. The interdependencies between the two networks are modeled by establishing the degree of connection through a spatial proximity criterion. The dependence of the WSS on the electricity grid stems from the need for power to operate the pumping stations, water treatment plants, and so forth. Having analyzed the networks in a case study the authors found that the water system is highly vulnerable to stoppages of the main elements in the electricity grid, which indicates that redundancy can be used to identify the weakest points in the networks.

\section{OPTIMIZATION MODELS}

Optimized decision models can be applied to WSSs to ensure low-cost solutions and high levels of performance and efficiency. So thought has to be given to which model will be best in given circumstances, according to the parameters available. Figure 2 characterizes the models that can be used.

The deterministic models used in recent decades ignore issues of risk and uncertainty and therefore does not consider the uncertainty of their parameters. 


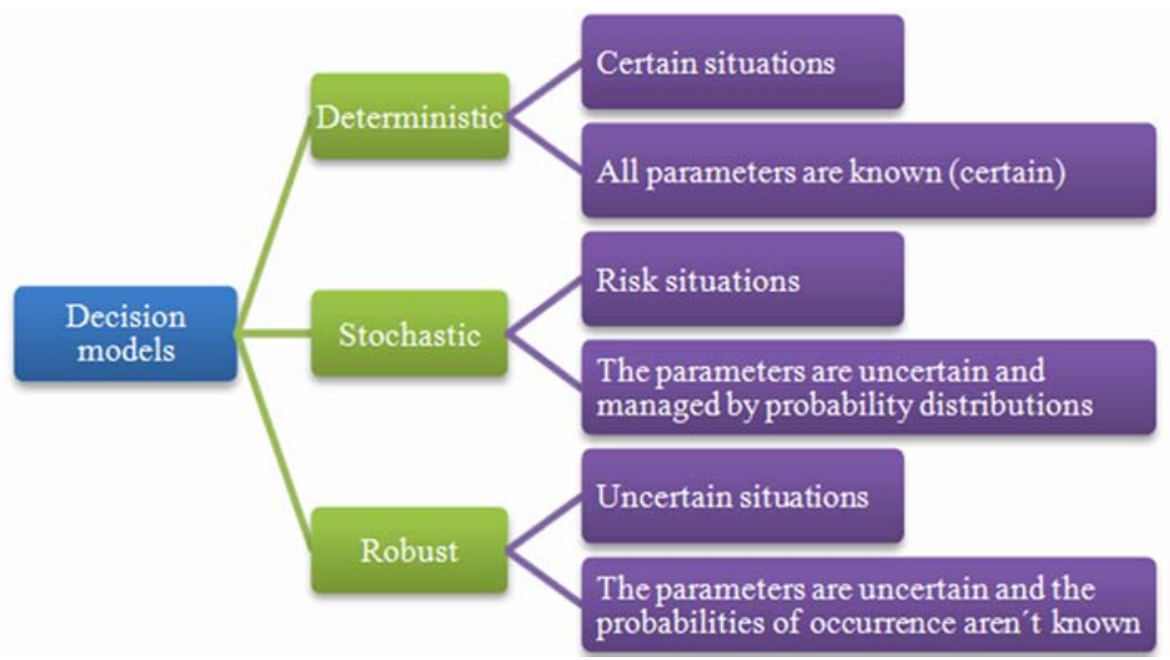

Figure 2: Decision models.

\section{ROBUST OPTIMIZATION}

Robust optimization models have sparked great interest in the scientific community and there is plentiful literature reporting the strides made in recent decades. This is a fairly new branch of the science and is still at the research stage.

Greenberg [20] defines robust optimization as a method that considers uncertainty and is similar to stochastic optimization in that it works with random variables. The difference between the methods is that stochastic optimization only considers parameters with well established probabilistic distribution, whereas robust models consider, in addition, parameters whose probabilistic distribution cannot be determined. Robust models have been considered in various areas of application as they may generate safer and more resilient solutions. Mulvey et al. [21] proposed the concept of robustness and presented a major synthesis of these methods, in which they give a theoretical introduction to them, and some applications.

In the theoretical introduction the authors look at two kinds of decision variables:

- $x$ : vector of the design decision variables whose optimum value is not conditioned by uncertain parameters;

- $y$ : vector of control decision variables which are subject to adjustment, depending not only on the value of the uncertain parameters but also on the optimum value of the $x$ variables.

The authors consider a generic robust optimization model given by:

$$
\min f\left(x, y_{1}, \ldots, y_{c}\right)+C_{p e n} p\left(e_{1}, \ldots, e_{c}\right)
$$

subject to:

$$
\begin{aligned}
A x & =b \\
H_{c} x+I_{c} y_{c}+e_{c} & =j_{c} \quad \forall c \in N C
\end{aligned}
$$




$$
x, y_{c} \geq 0 \quad \forall c \in N C
$$

where $A, b$ are fixed value parameters; $H_{c}, I_{c}, j_{c}$, uncertain value parameters of scenario $c ; N C$, number of scenarios; $e_{c}$, error vector of scenario $c$ that measure the infeasibility allowed by the control constraints (eqn 3 ); $f\left(x, y_{1}, \ldots, y_{c}\right)$, function relating different decision variables; $C_{\text {pen }}$, penalty coefficient used to find different solutions with differing robustness values and to compare them; and $p\left(e_{1}, \ldots, e_{c}\right)$ is penalty function used to penalize violations of the control constraints in some scenarios and to measure the model's robustness.

For the term $p\left(e_{1}, \ldots, e_{c}\right)$, and considering prob $_{c}$ the probability of occurrence of scenario $c$, the authors give two functions:

- $p\left(e_{1}, \ldots, e_{c}\right)=\sum_{c \in N C} \operatorname{prob}_{c} e_{c}^{T} e_{c}$ : a quadratic penalty function used on equality constraints when positive and negative constraint violations are considered;

- $p\left(e_{1}, \ldots, e_{c}\right)=\sum_{c \in N C} \operatorname{prob}_{c} \max \left\{0, e_{c}\right\}:$ a penalty function applied to inequality constraints when only positive violations are of interest.

The term that calculates the robustness of the model $f\left(x, y_{1}, \ldots, y_{c}\right)$ used in eqn (1) can be given by different functions. Considering $\varepsilon_{c}=k^{T} x+d_{c}^{T} y_{c}, k$ a vector of fixed value parameters and $d_{c}$ a vector of uncertain value parameters of scenario $c$, the authors give some possible functions:

$$
\begin{gathered}
f\left(x, y_{1}, \ldots, y_{c}\right)=\sum_{c \in N C} \operatorname{prob}_{c} \varepsilon_{c} \\
f\left(x, y_{1}, \ldots, y_{c}\right)=\sum_{c \in N C} \operatorname{prob}_{c} \varepsilon_{c}+C_{p e n} \sum_{c \in N C} \operatorname{prob}_{c}\left(\varepsilon_{c}-\sum_{c \in N C} \operatorname{prob}_{c} \varepsilon_{c}\right)^{2} \\
f\left(x, y_{1}, \ldots, y_{c}\right)=-\sum_{c \in N C} \operatorname{prob}_{c} U\left(\varepsilon_{c}\right)
\end{gathered}
$$

Equation (5) is the objective function of the stochastic optimization models and should be used only in low risk situations. Equation (6) can be used for medium and high decision risk situations and is called a mean/variance model, in which the risk value is equated to the variance value so that the risk can be controlled via a penalty parameter $C_{p e n}$ in the penalty factor (2nd term of the objective function). Distribution of the variable $\varepsilon_{c}$ needs to be symmetrical in relation to its mean value for this method can be used. Equation (7) can be used for high risk decision situations and it is based on the Von Neumann-Morgenstern utility curves in which, $U\left(\varepsilon_{c}\right)$ is a utility function. This method can be applied generally since it takes into account the probabilistic distributions of $\varepsilon_{c}$. Regarding the application of robust optimization the authors expose some cases studies. For reasons of space, all these cases and the others next to here were numbered for easy systematization at chapter 8 . The practical cases exposed by the authors are:

- power capacity expansion problem (I);

- matrix balancing problem (II);

- image reconstruction (III);

- airline allocation for the Air force: the STORM model (IV);

- scenario immunization (V);

- minimum weight structural design (VI). 
Snyder [22] also published an extensive literature review of infrastructure location problems resolved using stochastic and robust optimization. In the robust optimization models the author separates those composed of discrete parameters from others composed of continuous parameters since the uncertainties of the discrete parameters should be held to vary within a pre-established range of possible values. In addition, the author describes two of the commonest objectives used in these models, viz. the minimization of maximum costs and the minimization of maximum losses, in all possible scenarios accepted (MiniMax models).

Samsatli et al. [23] published a work on robust optimization models applied to engineering systems. The authors described mathematical techniques capable of capturing different aspects of risk and some robustness measures are defined through modifications of parameters and functions. According to the authors, two approaches are widely used to reformulate this model: probabilistic methodologies and methodologies based on scenario analysis. The latter is more usual. It requires the discretization of uncertain parameters to generate the scenarios and it gives a multi-scenario deterministic optimization problem. The scenarios can be determined explicitly if the combinations of parameters and associated probabilities are known a priori. By contrast, the scenarios may be generated implicitly assuming probability density function for the parameters and using an approximation to express the integral. The authors define robustness in terms of the variance of a measure of performance or through combinations of the mean and the variance and present some forms found in the literature. But they do not believe that variance is a sufficiently general measure of robustness, and so they give a general measure of robustness based on the violation of constraint. Based on that constraint, the authors show particular robustness measure, such as:

- linear penalty functions;

- Taguchi quality loss function;

- variance of constraints;

- constraints of signal-to-noise ratio;

- probability of constraint violation;

- expected constraint violation.

To reduce the complexity of the problem only the uncertain parameters that have most influence on the process in terms of magnitude and response type should be included. Furthermore, the combinatorial nature of the problem can be reduced by approximating the binary variables by continuous variables.

Two case studies were analyzed to apply these assumptions: the optimization of chemical reactors (VII) and the optimization of a fermentation process (VIII).

Suh and Lee [24] also proposed a robust optimization methodology for the planning and design of chemical processes. The general formula of the robust model described is similar to the stochastic model, but it has one extra constraint (eqn 12):

$$
\operatorname{Min} \sum_{c=1}^{N C} \operatorname{prob}_{c} C t_{c}
$$

subject to:

$$
\begin{gathered}
C t_{c}=f\left(x, y_{c}, \theta_{c}\right) \quad \forall c \in N C \\
h_{c}\left(x, y_{c}, \theta_{c}\right)=0 \quad \forall c \in N C
\end{gathered}
$$




$$
\begin{gathered}
g_{c}\left(x, y_{c}, \theta_{c}\right) \leq 0 \quad \forall c \in N C \\
R(C t) \leq R_{\lim }
\end{gathered}
$$

where $\operatorname{prob}_{c}$ is probability of occurrence of scenario $c ; C t_{c}$, costs in scenario $c$ calculated from function $f\left(x, y_{c}, \theta_{c}\right) ; x$, design variables vector; $y_{c}$, control variables vector for scenario $c$; $\theta_{c}$, uncertain parameters associated with scenario $c ; h_{c}\left(x, y_{c}, \theta_{c}\right)$, vectors of equality constraints on scenario $c$; $g_{c}\left(x, y_{c}, \theta_{c}\right)$, vectors of inequality constraints on scenario $c ; R(C t)$, measure of robustness as a function of cost; and $R_{\mathrm{lim}}$ is limit value of robustness measure.

By excluding the constraint (12) we get a stochastic optimization model. If, instead of the objective function (8), is used:

$$
\operatorname{Min}_{x, y_{c}} R(C t)
$$

The pure model of robust optimization achieved is so-called because it is a model whose sole purpose is to minimize a measure of robustness. For different values of $R_{\text {lim }}$ different cost solutions are obtained, and so a Pareto boundary can be constructed with pairs of optimal solution values. These solutions are given by resolving the stochastic model given by eqns (8)-(11), which determines the top limit of the robustness measure $R(C t)_{\text {stochastic }}$. Next the pure model of robust optimization is resolved (eqns (9)-(11) and (13)) to determine the lower limit of the robustness measure $R(C t)_{\text {robust }}$ Once these limits have been found, the multi-objective problem is resolved for values of $R_{\text {lim }}$ in the range:

$$
R(C t)_{\text {robust }} \leq R_{\text {lim }} \leq R(C t)_{\text {stochastic }}
$$

Finally, a model for designing a biological reactor for a wastewater treatment plant (IX) proposed by Afonso and Cunha [25] should be mentioned. In this model the robustness measure is given by the sum of the absolute value of the partial derivatives of performance in order of the parameters subject to uncertainty, so it is given as the sensitivity of the system to modifications of certain parameters.

\section{ROBUST OPTIMIZATION APPLIED TO WATER SUPPLY SYSTEMS}

Babayan et al. [26] solved the problem of the optimum robust design of WSSs using two methods, one based on redundancy of the system (X) and the other based on an integration method (XI). These authors consider the robustness of the system as the probability of simultaneously satisfying all the pressure constraints that is, guaranteeing nodal pressures above a pre-set figure, for all the system's nodes. The sources of uncertainty considered in these methods were the nodal consumption and the coefficients of roughness of the pipes.

Cunha and Sousa [27] presented a decision model for designing a WSS (XII) based on the concept of robust optimization and resolved by means of a simulated annealing algorithm. Through this model the authors show, for a network subject to different consumption scenarios, the increased costs associated with gains in robustness for different values of a penalty coefficient. This makes it possible to give decision makers cost-robustness ratios to help them choose which decision to adopt.

For the robust management of WSSs, Jeong et al. [28] propose a mathematical model capable of identifying a WSS's vulnerabilities, the optimal allocation of available security resources and the reduction of the consequences of pre-planned terrorist attacks. The authors describe a model to control, or determine a suitable way to operate, a WSS damaged by terrorist attack (XIII). Terrorist attacks may be chemical, biological, cyber or physical, but this article focuses 
on physical attacks because they are the most likely on account of the low level of expertise required to mount them. After an attack, just one part of the system is assumed to remain functional. The mitigation model developed by the authors allows the consequences of a cut to the water supply to be reduced by means of ascribing a priority level to each consumption node of the network.

Carr et al. [29] report models used for the robust optimal placement of sensors in a WSS to detect contaminants accidentally or criminally injected into a particular network node (XIV). Here it is assumed that all the points downstream of the contaminated node are affected. The authors describe two kinds of decision models: the expected reduction of network contamination as defined by the number of contaminated nodes and the minimization of the number of people exposed to contamination.

\section{SYSTEMATIZATION}

A detailed analysis of the works mentioned has made it possible to establish the following modes of including robustness in the specified optimization models:

- Multi-scenario analyses: the decision model is resolved for a series of scenarios, i.e. for different values of uncertain parameters, so that the global solution obtained functions properly for all the scenarios tested.

- Parameter variance: in the object function of the model, a term is implemented to calculate the variance of a particular uncertain parameter.

- Penalty factors: constant parameters of the model normally used to penalize constraint violations, and through their variation, to find different possible solutions with different robustness values.

- Utility functions: determine the benefits of a particular process according to the satisfaction of the objectives achieved.

- Security factors: the uncertain parameters of the model are increased so that the final solution will function well, even in adverse circumstances. The larger these factors the greater the robustness of the final solution and the higher the cost of the solution.

- Integration method: used to calculate the mean and standard deviation of the uncertain parameters so as to arrive at values that can be used as security factors in the control constraints.

- Performance variation: established by the derivative of a performance measure of the system in order of the different uncertain parameters of the model which conveys the sensitivity of the system to parameter modification; limiting superiorly this derivative is to limit the variation of system performance and with this, impose a minimum level of robustness.

- Constraint violations: used to quantify the error in parameters determined by solving the model and the target values that should have been obtained; (in the case of WSSs, a common constraint of this type is the violation of minimum admissible pressure at various nodes of the system);

- MiniMax: models which use objective functions that aim to reduce the maxima of certain parameters in all the possible scenarios so that the solution arrived, functions even in the worst possible parameter situations.

Table 1 has been constructed to summarize the modes of including robustness in the optimization models presented in this paper. The different robustness modes used can be checked quite quickly.

\section{CONCLUSIONS}

This paper has characterized the vulnerabilities, risks, interdependencies with other infrastructures and optimization models normally applied to WSSs. It then described the state of the art of robust 


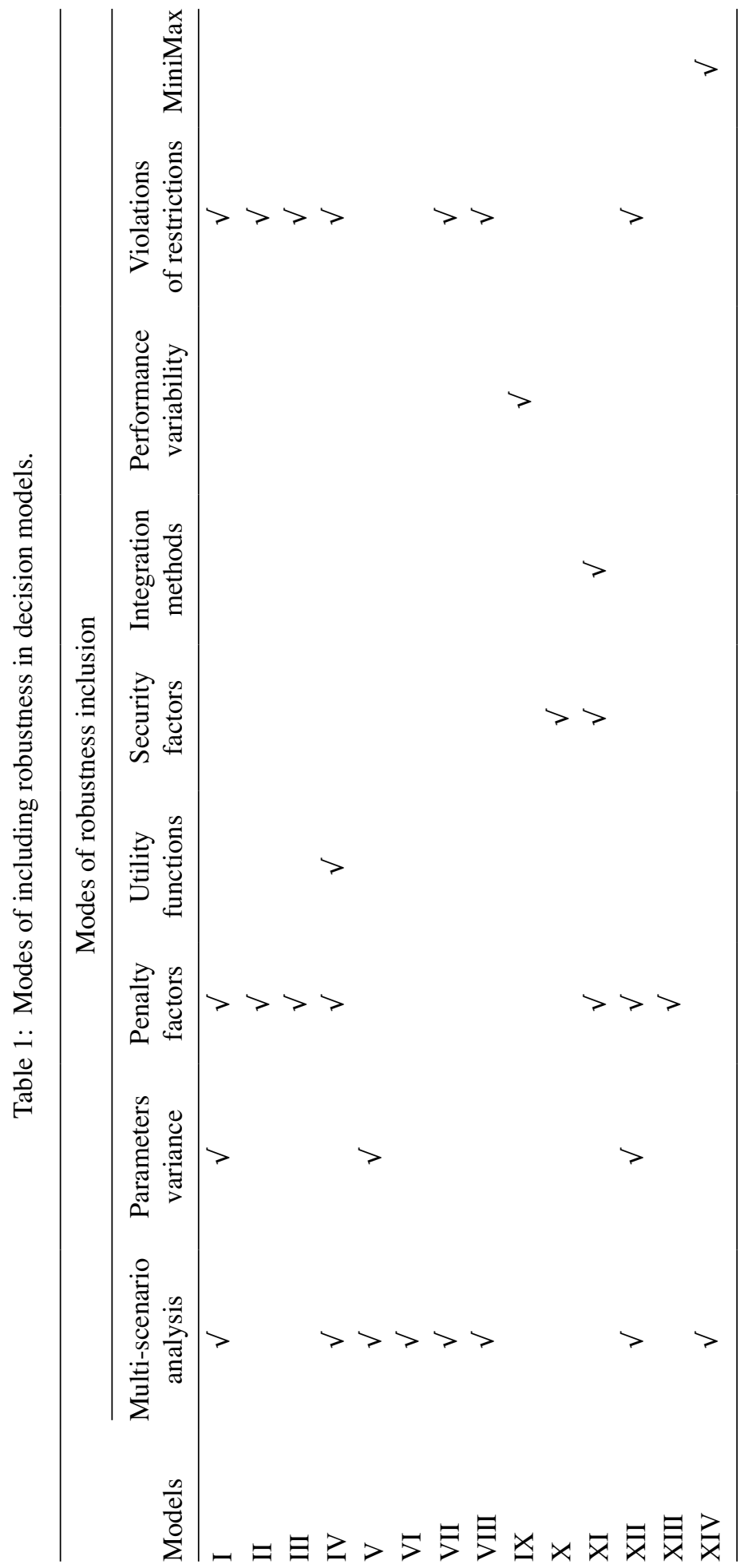


optimization models applied to diverse engineering systems. Finally, it presented a systematization of the different modes found in the case study literature on the inclusion of robustness in decision models. This literature review has enabled some conclusions to be drawn, as set forth below:

- WSSs are highly vulnerable because of their great exposure, extent and accessibility.

- There is a huge number of extreme adverse situations, provoked by natural events or human activity, that can affect WSSs.

- Risk management in WSSs requires a systematic process of analysis, assessment and control.

- WSSs rely on other infrastructures, which means that a failure in one of these systems is linked to an interruption of services provided by other infrastructure networks.

- A robust solution of a model is one which remains 'close' to the optimum for any values of uncertain parameters.

- Robust optimization can be applied to many areas of science to find more 'secure' solutions.

- When designing WSSs, robust optimization methods lead to solutions that function satisfactorily, even under extreme operating conditions.

- The main forms of including robustness in the optimization models found in the literature are multi-scenario analysis, penalty factors and constraint violations.

\section{ACKNOWLEDGMENT}

This work was carried out under a project funded by the Foundation for Science and Technology (FCT): 'Gestão integrada do risco de infraestruturas públicas: os sistemas de abastecimento de água' (Integrated risk management of public infrastructures: water supply systems) (PTDC/ECM/64821/2006).

\section{REFERENCES}

[1] Parfomak, P.W., Guarding America: Security Guards and U.S. Critical Infrastructure Protection, Congressional Research Service. CRS Report for Congress, 2004.

[2] Haimes, Y.Y., Sustainable operation of threatened infrastructures. Journal of Infrastructure Systems, ASCE, Viewpoint, 4(1), pp. 1-4, 1998.

[3] Danneels, J.J. \& Finley, R.E., Assessing the vulnerabilities of U.S. drinking water systems. Journal of Contemporary Water Research and Education, 129(1), pp. 8-12, 2004. doi:10.1111/ j.1936-704X.2004.mp129001003.x

[4] Haimes, Y.Y., Matalas, N.C., Lambert, J.H., Jackson, B.A. \& Fellows, J.F.R., Reducing vulnerability of water supply systems to attack. Journal of Infrastructure Systems, ASCE, 4(4), pp. 164-177, 1998. doi:10.1061/(ASCE)1076-0342(1998)4:4(164)

[5] Matalas, N.C., Acts of nature and potential acts of terrorists: contrast relative to water resource systems. Journal of Water Resources Planning and Management, ASCE, Editorial, 131(2), pp. 79-80, 2005.

[6] Viera, J.M.P., Valente, J.C.T., Peixoto, F.M.S.P.M. \& Morais, C.M.G.D., Elaboração e implementação de planos de contingência em sistemas de abastecimento de águas. Associação Portuguesa dos Recursos Hídricos, $8^{\circ}$ Congresso da água, 2006 (in Portuguese).

[7] Meinhardt, P.L., Water and bioterrorism: preparing for the potential threat to U.S. water supplies and public health. Annual Review Public Health, 26, pp. 213-237, 2005. doi:10.1146/ annurev.publhealth.24.100901.140910

[8] ICE (Institution of Civil Engineers), Megacities: Reducing Vulnerability to Natural Disaster, Thomas Telford Services Ltd., 1995.

[9] WHO (World Wealth Organization), Natural Disaster Mitigation in Drinking Water and Sewerage Systems - Guidelines for Vulnerability Analysis, PAHO: Washington, DC, p. 110, 1998.

[10] OFPP (Office Fédéral de la Protection de la Population), Catastrophes et situation d'urgence en Suisse: une appréciation des risques du point de vue de la protection de la population. Berne, Office fédéral de la protection de la population, 2003. 
[11] Ezell, B.C., Farr, J.V. \& Wiese, I., Infrastructure risk analysis model. Journal of Infrastructure Systems, 6(3), pp. 114-117, 2000. doi:10.1061/(ASCE)1076-0342(2000)6:3(114)

[12] Ezell, B.C., Farr, J.V. \& Wiese, I., Infrastructure risk analysis of municipal water distribution system. Journal of Infrastructure Systems, 6(3), pp. 118-122, 2000. doi:10.1061/(ASCE)10760342(2000)6:3(118)

[13] Tidwell, V.C., Cooper, J.A. \& Silva, C.J., Threat assessment of water supply systems using Markov latent effects modelling. Journal of Water Resources Planning and Management, ASCE, 131(3), pp. 218-227, 2005. doi:10.1061/(ASCE)0733-9496(2005)131:3(218)

[14] Kunreuther, H., Cyr, C., Grossi, P. \& Tao, W., Using Cost-Benefit Analysis to Evaluate Mitigation for Lifeline Systems, 2002 [Online]. Available from: http://opim.wharton. upenn.edu/risk/downloads/01-14-HK.pdf [Accessed 1 October 2009].

[15] Chang, S.E., Evaluating disaster mitigations: methodology for urban infrastructure systems. Natural Hazards Review, 4(4), pp. 186-196, 2003. doi:10.1061/(ASCE)1527-6988(2003)4:4(186)

[16] Haimes, Y.Y., Infrastructure interdependencies and homeland security. Journal of Infrastructure Systems, ASCE, Editorial, 11(2), pp. 65-66, 2005.

[17] Haimes, Y.Y. \& Horowitz, B.M., Modelling interdependent infrastructures for sustainable counterterrorism. Journal of Infrastructure Systems, ASCE, Forum, 10(2), pp. 33-42, 2004.

[18] Haimes, Y.Y. \& Jiang, P., Leontief-based model of risk in complex interconnected infrastructures. Journal of Infrastructure Systems, ASCE, 7(1), pp. 1-12, 2001. doi:10.1061/(ASCE)10760342(2001)7:1(1)

[19] Osorio, L.D., Craig, J.I., Goodno, B.J. \& Bostrom, A., Interdependent response of networked systems. Journal of Infrastructure Systems, ASCE, 13(3), pp. 185-194, 2007. doi:10.1061/ (ASCE)1076-0342(2007)13:3(185)

[20] Greenberg, H.J., Mathematical programming glossary. Informs Computing Society, 2008.

[21] Mulvey, M.M., Vanderbei, R.J. \& Zenios, S.A., Robust optimization of large-scale systems. Operations Research, 43(2), pp. 264-281, 1995. doi:10.1287/opre.43.2.264

[22] Snyder, L.V., Facility location under uncertainty: a review. IIE Transactions, 38(7), pp. 547-564, 2006. doi:10.1080/07408170500216480

[23] Samsatli, N.J., Papageorgiou, L.G. \& Shah, N., Robustness metrics for dynamic optimization models under parameter uncertainty. Process Systems Engineering AIChE Journal, 44(9), pp. 1993-2006, 1998.

[24] Suh, M. \& Lee, T.Y., Robust optimization method for the economic term in chemical process design and planning. American Chemical Society, 40(25), pp. 5950-5959, 2001.

[25] Afonso, P.M. \& Cunha, M.C., Robust optimal design of activated sludge bioreactors. Journal of Environmental Engineering, ASCE, 133(1), pp. 44-52, 2007. doi:10.1061/(ASCE)07339372(2007)133:1(44)

[26] Babayan, A.V., Savic, D.A., Walters, G.A. \& Kapelan Z.S., Robust least-cost design of water distribution networks using redundancy and integration-based methodologies. Journal of Water Resources Planning and Management, ASCE, 133(1), pp. 67-77, 2007.

[27] Cunha, M.C. \& Sousa, J., Dimensionamento Robusto de Sistemas de Abastecimento de Distribuição de Água. VIII Seminário Ibero-Americano, 2008 (in Portuguese).

[28] Jeong, H.S., Qiao, J., Abraham, D.M., Lawley, M., Richard, J.P. \& Yih, Y., Minimizing the consequences of intentional attack on water infrastructure. Computer Aided Civil and Infrastructure Engineering, 21(2), pp. 79-92, 2006. doi:10.1111/j.1467-8667.2005.00419.x

[29] Carr, R.D., Greenberg, H.J., Hart, W.E., Lauer, G.K.E., Lin, H., Morrison, T. \& Phil ips, C.A., Robust optimization of contaminant sensor placement for community water systems. Mathematical Programming: Series A and B, 107(1), pp. 337-356, 2005. 